\title{
Once malaria is eliminated, more attention should be paid to imported malaria: Data from five years of surveillance in the City of Yiwu in eastern China
}

\author{
Xuanjun Dong ${ }^{1, \S}$, Jie Yang ${ }^{1, \S}$, Lianqin Lou ${ }^{2}$, Liebo Zhu ${ }^{1}$, Xiayan Feng ${ }^{1}$, Linong Yao,* \\ ${ }^{1}$ Yiwu Center for Disease Control and Prevention, Yiwu, Zhejiang, China; \\ ${ }^{2}$ Yiwu Central Hospital, Yiwu, Zhejiang, China; \\ ${ }^{3}$ Zhejiang Provincial Center for Disease Control and Prevention, Hangzhou, Zhejiang, China.
}

\begin{abstract}
Summary This study reviewed and analyzed data on malaria cases in Yiwu from 2012 to 2016 via a webbased system for managing and reporting information on infectious diseases. A total of 161 cases were diagnosed $(77.02 \%$ due to Plasmodium falciparum, $18.01 \%$ due to $P$. vivax, $4.35 \%$ due to P. ovale, and $\mathbf{0 . 6 2 \%}$ due to P. malariae). One case was imported from Yunnan Province in China and the others were imported from overseas. The ratio of male to female patients was 7.47:1. The average age was 36.34 years (SD: 9.63). Most cases $(87.58 \%)$ were imported from 1 of 30 countries in Africa. As malaria is gradually being eliminated in China, the main task at this stage has transitioned to the prevention and control of cases of imported malaria. Particular attention should be paid to malaria cases from Africa.
\end{abstract}

Keywords: Malaria, imported, China

\section{Introduction}

Malaria is a debilitating parasitic disease. According to the Roll Back Malaria (RBM) Partnership, each year approximately 860,000 people $(89 \%$ in the African region, followed by $6 \%$ in the Eastern Mediterranean region, and 5\% in the South-East Asia region), mainly children and women, succumb to the disease. Despite successful elimination programs, a resurgence of malaria has occurred in Eastern Europe, Jamaica, the Bahamas $(1,2)$, and the Korean peninsula $(3,4)$. The City of Yiwu had been an area of China with a high rate of malaria transmission, with a morbidity of 2,830/10,000 in 1957. Due to large-scale surveys and anti-malaria campaigns, the incidence of malaria dropped sharply below 1/10,000 after 1977, and the last autochthonous case was reported in June 2008. With the growth of international trade and travel, migrants have accounted for most cases

Released online in J-STAGE as advance publication June 18, 2017.

${ }^{\S}$ These authors contributed equally to this work.

*Address correspondence to:

Dr. Linong Yao, Zhejiang Provincial Center for Disease Control and Prevention, Hangzhou, Zhejiang, China.

E-mail: ylinong@163.com of imported malaria in Yiwu. Given the high rate of transmission and the susceptibility of the population, effective strategies are required to prevent re-introduction and re-establishment of the disease in Yiwu.

The current study reviewed and analyzed data on malaria cases in Yiwu from 2012 to 2016 via a webbased system for managing and reporting information on infectious diseases. Cases must be differentiated into autochthonous and imported cases in line with the "13-7" strategy (which refers to reporting suspected cases of malaria within 1 day, investigating and confirming the diagnosis within 3 days, and investigating the focus and responding to prevent further transmission within 7 days) launched in 2012 (5). Clinically diagnosed cases, laboratory confirmed cases, autochthonous cases, and imported cases were all included in this study. Population data were obtained from the Bureau of Statistics of Yiwu. Spatial cluster analysis was performed to test whether the malaria cases were randomly distributed spatially; if not, any identified spatial clusters of disease were examined for statistical significance. Statistics were described using the software Excel version 2010.

From 2012 to 2016, a total of 161 cases of malaria were reported in Yiwu, and the annual average incidence was 13.05/10,000 during this time. There were 22 cases in 2012, 34 in 2013, 29 in 2014, 30 in 2015, and 46 in 
2016. As a result of proper treatment, no deaths occurred. The ratio of male to female patients was 7.47:1, and the average age was $36.34 \pm 9.63$ years. All of the cases were imported from abroad, except for one imported from Yunnan Province. More than half of the patients were foreigners $(55.90 \%)$. Most patients were in the service industry $(83.85 \%)$ or migrant workers $(11.18 \%)$ and lived on Choucheng Street $(55.76 \%)$ or Jiangdong Street $(24.22 \%)$ (Table 1$)$. More than three quarters (77.02\%) of the cases were due to P. falciparum, 29 cases (18.01\%) were due to P. vivax, 7 cases $(4.35 \%)$ were due to $\mathrm{P}$. ovale, and 1 case $(0.62 \%)$ was due to P. malariae (Table 2). Five cases of P. vivax and 1 case of P. ovale recurred. Twenty-five cases $(15.53 \%)$ were imported in August. The seasonal distribution of cases did not differ significantly throughout the year. Most cases (87.58\%) were imported from 1 of 30 countries in Africa, and the three leading countries were Ghana, Nigeria, and Angola. Sixteen cases were from South Asia, and 10 of those were from India (Table 3). The time interval from onset to diagnosis was 0-47 days, with a median of 3 days. Most cases (79.5\%) were diagnosed 5 days after

Table 1. Characteristics of patients with imported malaria in Yiwu from

\begin{tabular}{lrc}
\hline Characteristics & Cases & Proportion (\%) \\
\hline Gender & & \\
Male & 142 & 88.20 \\
Female & 19 & 11.80 \\
Nationality & & \\
$\quad$ Chinese & 71 & 44.10 \\
Foreigner & 90 & 55.90 \\
Occupation & & \\
$\quad$ Service industry & 135 & 83.85 \\
$\quad$ Migrant worker & 18 & 11.18 \\
$\quad$ Other & 8 & 4.97 \\
Present address & & \\
$\quad$ Choucheng Street & 93 & 57.76 \\
$\quad$ Jiangdong Street & 39 & 24.22 \\
$\quad$ Beiyuan Street & 7 & 4.35 \\
Other & 22 & 13.67 \\
Total & 161 & \\
\hline
\end{tabular}

onset. One hundred and fourteen cases of malaria were diagnosed at primary care facilities. Eighty-one patients had previously had malaria.

This study revealed substantial changes in the epidemiological characteristics of malaria reported in Yiwu from 2012 to 2016. Yiwu faces an increasing incidence of malaria imported by migrants returning from Africa and South Asia, and an epidemic may occur if no efforts are made to prevent these outbreaks. The international flow of people has led to a large number of cases of imported malaria. The "1-3-7" strategy for elimination of malaria should be followed and all of the indicators and timeframes must be observed to promptly diagnosis and treat the increasing number of cases of imported malaria in Yiwu. Potential reservoirs must be eliminated and outbreaks due to imported pathogens must be prevented. The government should continue to emphasize the elimination of malaria and ensure efforts are funded. Effective mechanisms of multisectoral cooperation and coordination should be enhanced. Capacity should also be improved with a focus on surveillance, urgent responses, diagnosis, and treatment. Moreover, studies by and cooperation among universities, research institutes, and government agencies should be fostered and enhanced as malaria is being eliminated.

\section{Acknowledgements}

The authors with to thank all of the staff members at the Yiwu CDC. This study was supported by the Zhejiang Provincial Health Department (Grant No. 2015ZHB016) and the Yiwu Science and Technology Bureau Program (Grant No. 14-3-06).

\section{References}

1. WHO. World Malaria Report 2009. Geneva: World Health Organization; 2009. http://www.who.int/malaria/ world_malaria_report_2009/mal2009_rep_chap5_0040.

Table 2. Classification of imported malaria in Yiwu from 2012-2016

\begin{tabular}{|c|c|c|c|c|c|c|c|}
\hline Type & 2012 & 2013 & 2014 & 2015 & 2016 & Total & Proportion $(\%)$ \\
\hline P. falciparum & 17 & 21 & 21 & 25 & 40 & 124 & 77.02 \\
\hline Plasmodium vivax & 5 & 11 & 6 & 3 & 4 & 29 & 18.01 \\
\hline P. ovale & 0 & 2 & 1 & 2 & 2 & 7 & 4.35 \\
\hline P. malariae & 0 & 0 & 1 & 0 & 0 & 1 & 0.62 \\
\hline Total & 22 & 34 & 29 & 30 & 46 & 161 & 100 \\
\hline
\end{tabular}

Table 3. Origin of imported malaria in Yiwu from 2012-2016

\begin{tabular}{lccccc}
\hline Type & Africa & South Asia & Southeast Asia & Oceania & China \\
\hline P. falciparum & 123 & 0 & 0 & 0 & 1 \\
P. vivax & 10 & 16 & 2 & 1 & 0 \\
P. ovale & 7 & 0 & 0 & 0 & 0 \\
P. malariae & 1 & 0 & 0 & 0 & 0 \\
Total & 141 & 16 & 2 & 1 & 1 \\
Proportion (\%) & 87.58 & 9.94 & 1.24 & 0.62 & 1 \\
\hline
\end{tabular}


$p d f ? u a=1$. (Accessed May 12, 2017).

2. Webster-Kerr K, Peter Figueroa J, Weir PL, Lewis-Bell K, Baker E, Horner-Bryce J, Lewis-Fuller E, Bullock Ducasse M, Carter KH, Campbell-Forrester S. Success in controlling a major outbreak of malaria because of Plasmodium falciparum in Jamaica. Trop Med Int Health. 2011; 16:298306.

3. Kho WG, Jang JY, Hong ST, Lee HW, Lee WJ, Lee JS. Border malaria characters of re-emerging Plasmodium vivax in the Republic of Korea. Korean J Parasitol. 1999; 37:71-76.

4. Global Health Science Group. Eliminating malaria in the Republic of Korea, Country briefing; 2015. https:// globalhealthsciences.ucsf.edu/sites/globalhealthsciences. ucsf.edu/files/pub/rokorea-2015-final.pdf. (Accessed May 12, 2017).

5. Cao J, Sturrock HJ, Cotter C, Zhou S, Zhou H, Liu Y, Tang L, Gosling RD, Feachem RG, Gao Q. Communicating and monitoring surveillance and response activities for malaria elimination: China's "1-3-7" strategy. PLoS Med. 2014; 11:e1001642.

(Received May 17, 2017; Revised June 12, 2017; Accepted June 14, 2017) 